\title{
Sample Entropy Approach to the Examination of Cardio-Respiratory Coupling in Response to Cardiac Resynchronization Therapy ${ }^{\dagger}$
}

\author{
Mirjana M Platiša ${ }^{1}$, Nikola N Radovanović ${ }^{2}$, Goran Milašinović ${ }^{2,3}$ and Siniša U Pavlović ${ }^{2,3}$ \\ 1 Institute of Biophysics, Faculty of Medicine, Belgrade University, Belgrade, Serbia \\ 2 Pacemaker Center, Clinical Center of Serbia, Belgrade, Serbia \\ 3 Faculty of Medicine, Belgrade University, Belgrade, Serbia \\ † Presented at the Entropy 2021: The Scientific Tool of the 21st Century, 5-7 May 2021; Available online: \\ https://sciforum.net/conference/Entropy2021/.
}

Published: 5 May 2021

Cardiac resynchronization therapy (CRT) is a well-established therapy for symptomatic patients with heart failure and reduced left ventricular ejection fraction. It is known that patients with heart failure have altered cardio-respiratory interactions, but it has not been examined whether resynchronization therapy leads to changes in coupling of cardiac and respiratory rhythm, and whether the success of this therapy leads to restoring of cardio-respiratory interactions. In these patients, in addition to sinus rhythm, different types of arrhythmias usually appear, which limits the application of linear methods in analysis of interbeat interval time series. Therefore they should be analyzed with non-linear techniques and we applied the sample entropy approach.

Twenty minutes of ECG (RR intervals) and respiratory signal were recorded simultaneously in 47 patients with heart failure and CRT indication. The interbeat interval time series in patients with sinus rhythm, sinus rhythm with ventricular extrasystoles and with atrial fibrillation were analyzed. Sample entropy values were calculated from RR interval time series (SampEnRR) and respiratory signal time series (SampEnResp) to assess their complexity/regularity as well as cross sample entropy (CrossSampEn) to estimate their asynchrony. Measurements were performed before (baseline) and approximately 9 months after CRT implantation (follow-up). After follow-up, patients were divided into two groups, responders and non-responders, in relation to the response to CRT, which was assessed according to changes in certain clinical parameters.

In both groups, there was no difference in SampEnRR between baseline and follow-up. However, in the non-responders group, a significant increase was obtained in SampEnResp and CrossSampEn ( $p$ $<0.05)$. Responders to CRT showed significant decrease in heart rate and breathing frequency, while non-responders showed only a significant decrease in heart rate. Our results indicate that in nonresponders to CRT, respiratory rhythm is not adapting to changes in cardiac dynamics, which resulted in loss of their synchrony.

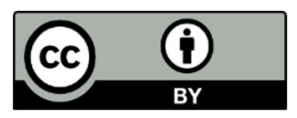

(C) 2021 by the authors. Licensee MDPI, Basel, Switzerland. This article is an open access article distributed under the terms and conditions of the Creative Commons Attribution (CC BY) license (http://creativecommons.org/licenses/by/4.0/). 